\title{
Long actin-based cellular protrusions as novel evidence of the cytopathic effect induced in immune cells infected by the ectromelia virus
}

\author{
LIDIA SZULC-DĄBROWSKA ${ }^{1}$, KAROLINA P. GREGORCZYK-ZBOROCH \\ JUSTYNA STRUZIK', ZBIGNIEW WYŻEWSKI ${ }^{1}$, AGNIESZKA OSTROWSKA ${ }^{2}$, \\ FELIX N. TOKA ${ }^{1,3}$, MAEGORZATA GIERYŃSKA ${ }^{l}$
}

${ }^{1}$ Department of Preclinical Sciences, Faculty of Veterinary Medicine, Warsaw University of Life Sciences, Warsaw, Poland ${ }^{2}$ Analytical Center, Warsaw University of Life Sciences, Warsaw, Poland

${ }^{3}$ Department of Biomedical Sciences, Ross University School of Veterinary Medicine, St. Kitts \& Nevis, West Indies

\begin{abstract}
The aim of the study was to evaluate the influence of ectromelia virus (ECTV) infection on actin cytoskeleton rearrangement in immune cells, such as macrophages and dendritic cells (DCs). Using scanning electron and fluorescence microscopy analysis we observed the presence of long actin-based cellular extensions, formed by both types of immune cells at later stages of infection with ECTV. Such extensions contained straight tubulin filaments and numerous punctuate mitochondria. Moreover, these long cellular projections extended to a certain length and formed convex structures termed "cytoplasmic packets". These structures contained numerous viral particles and presumably were sites of progeny virions' release via budding. Further, discrete mitochondria and separated tubulin filaments that formed a scaffold for accumulated mitochondria were visible within cytoplasmic packets. ECTV-induced long actin-based protrusions resemble "cytoplasmic corridors" and probably participate in virus dissemination. Our data demonstrate the incredible capacity for adaptation of ECTV to its natural host immune cells, in which it can survive, replicate and induce effective mechanisms for viral spread and dissemination.
\end{abstract}

Key words: ectromelia virus, cellular protrusions, macrophages, dendritic cells.

(Centr Eur J Immunol 2018; 43 (4): 363-370)

\section{Introduction}

The actin cytoskeleton plays an indispensable role in viral replication cycle. Stimulation of actin filament remodeling takes place during virion attachment and endocytosis, viral replication and assembly, and viral egress and spread. To spread successfully from cell to cell, viruses interact with actin to promote budding and/or production of different types of cellular extensions for directional, long-distance transport of progeny virions [1].

Ectromelia virus (ECTV), an orthopoxvirus, which is a causative agent of mousepox, has been used extensively as a model of smallpox, caused by variola virus (VARV), due to many similarities shared by both viruses [2]. It is assumed that the cessation of vaccination against smallpox may contribute to increased risk of zoonotic infections in humans caused by other orthopoxviruses, such as monkeypox (MPXV) and cowpox (CPXV) viruses [3]. Moreover, both VARV and MPXV are considered threat agents that can be used as bioterrorist weapons [4]. Additionally, in- creased incidents of outbreaks caused by orthopoxviruses in domestic animals have also been reported [5]. Therefore, a better understanding of the interactions between the virus and its natural host should assist in developing new strategies for antiviral therapy.

Previous studies have demonstrated that in fibroblasts and epithelial cells, ECTV induces formation of short (actin tails, "dendrites") and/or long [tunneling nanotubes (TNT), cytoplasmic corridors] actin-based cellular projections for efficient intercellular spread in vitro [6-8]. However, it is not known how orthopoxviruses influence actin cytoskeleton of immune cells, especially antigen presenting cells (APCs), such as dendritic cells (DCs) and macrophages. Therefore, we designed experiments to address the impact of ECTV on the cytoskeleton of murine macrophages and DCs. The results show that long actin-based cellular extensions develop following infection of antigen presenting cells with ECTV, an observation that has not been reported before.

Correspondence: Lidia Szulc-Dąbrowska, PhD, Department of Preclinical Sciences, Faculty of Veterinary Medicine,

Warsaw University of Life Sciences, 8 Ciszewskiego St., 02-786 Warsaw, Poland, e-mail: lidia_szulc@sggw.pl

Submitted: 12.05.2017; Accepted: 5.07.2017 


\section{Material and methods}

\section{Mice}

Inbred, male BALB/c (H-2 $\left.{ }^{\mathrm{d}}\right)$ 8-12 week old mice were used in the study. All mice were purchased from the animal facility at Maria Skłodowska-Curie Memorial Cancer Centre and Institute of Oncology in Warsaw, Poland. All experiments were carried out in accordance with institutional Guidelines for Care and Use of Laboratory Animals and were approved by the $3^{\text {rd }}$ Ethical Committee for Animal Experimentation at Warsaw University of Life Sciences - SGGW (permit no. 34/2012).

\section{Cell cultures}

Mouse monocyte/macrophage RAW 264.7 (TIB-71) cell line, transformed with Abelson murine leukemia virus, was obtained from the American Type Culture Collection (ATCC). Cells were cultured in the Glutamax-I RPMI 1640 medium (Gibco BRL) supplemented with $10 \%$ heatinactivated fetal bovine serum (FBS; Sigma-Aldrich) and $1 \%$ antibiotic solution (100 U/ml penicillin, $100 \mu \mathrm{g} / \mathrm{ml}$ streptomycin; Sigma-Aldrich) at $37^{\circ} \mathrm{C}$ in a humidified $5 \% \mathrm{CO}_{2}$ atmosphere. The cells were passaged twice a week and were rigorously tested for mycoplasma contamination using DNA-specific fluorescent Hoechst 33258 [9]. All cell cultures were mycoplasma-free.

GM-CSF-derived bone marrow cells (GM-BM) were obtained as previously described $[10,11]$ with minor modifications. Briefly, femurs and tibiae were removed from mice $(n=4)$ and trimmed of muscle tissue. After disinfection in $70 \%$ alcohol, ends of bones were cut and the marrow was flushed. After vigorous pipetting, bone marrow cells were cultured in 6-well plates in Glutamax-I RPMI-1640 medium supplemented with $10 \%$ heat-inactivated FBS, $1 \%$ antibiotic solution, $50 \mu \mathrm{M}$ 2-mercaptoethanol (Sigma-Aldrich) and $20 \mathrm{ng} / \mathrm{ml}$ recombinant mouse granulocyte-macrophage colony stimulating factor (rmGM-CSF, R\&D Systems). After 8 days of culture at $37^{\circ} \mathrm{C}$ in a humidified $5 \% \mathrm{CO}_{2}$ atmosphere, GM-BM were enriched using MACS CD11 $\mathrm{c}^{+}$labeled magnetic beads (Miltenyi Biotec) [11].

\section{Virus}

Highly infectious Moscow strain of ECTV (ATCC VR-1374) was propagated and titrated by the plaque-forming units (PFU) method in the Vero cell line (ATCC CCL81). Briefly, cells were infected with ECTV at a multiplicity of infection $(\mathrm{MOI})=0.01$ and incubated at $37^{\circ} \mathrm{C}$ in a humidified $5 \% \mathrm{CO}_{2}$ atmosphere until the cytopathic effect was prominent. Infected cells were then harvested and lysed during repeated freeze-thaw cycles. After purification, virus stocks were stored in aliquots at $-70^{\circ} \mathrm{C}$ until used. Titration of ECTV stocks was performed by serial dilutions in Vero cell culture. The virus titer was $4 \times 10^{8}$ $\mathrm{PFU} / \mathrm{ml}$.
RAW 264.7 cells and GM-BM, seeded on microscopic slides placed in a 24-well plate at a density of $2 \times 10^{5}$ cells/well, were infected with ECTV at MOI $=1$. After $60 \mathrm{~min}$ of viral adsorption at $37^{\circ} \mathrm{C}$, the cells were incubated at $37^{\circ} \mathrm{C}$ in a humidified $5 \% \mathrm{CO}_{2}$ atmosphere for an additional 24 hours. Control (uninfected) cultures were identically processed but not infected with ECTV.

\section{May-Grünwald-Giemsa staining}

The May-Grünwald-Giemsa (MGG) staining method was used for cell morphology assessment. Mock- or ECTV-infected RAW 264.7 cells, cultured on microscope slides for $24 \mathrm{~h}$, were fixed in methanol for $5 \mathrm{~min}$ and stained with May-Grünwald solution (Sigma-Aldrich) for $5 \mathrm{~min}$ and Giemsa solution (Sigma-Aldrich) diluted $1: 20$ for $20 \mathrm{~min}$. After rinsing in deionized water, slides were air dried and observed under the inverted microscope Olympus IX71 (Olympus). Images were recorded with the $40 \times$ objective.

\section{Immunofluorescence staining}

Immunofluorescence staining was performed as previously described [8]. Briefly, cells were fixed either with ice-cold absolute methanol (Avantor Performance Materials Poland) for 2 min or $4 \%$ paraformaldehyde (PFA, Sigma-Aldrich) in PBS for $20 \mathrm{~min}$. In some experiments, before fixation, the mitochondria were stained with 300 nM MitoRed dye (cat. no. 53271; Sigma-Aldrich) for $20 \mathrm{~min}$ at $37^{\circ} \mathrm{C}$ in a humidified atmosphere of $5 \% \mathrm{CO}_{2}$ in the air. Then cells were permeabilized with $0.5 \%$ Triton X-100 (Sigma-Aldrich) in PBS and blocked with 3\% bovine serum albumin (BSA, Sigma-Aldrich) in $0.1 \%$ Triton X-100 in PBS. F-actin was labeled with either tetramethylrhodamine isothiocyanate (TRITC)- or fluorescein isothiocyanate (FITC)-conjugated phalloidin (cat. no. P1951 and P5282; Sigma-Aldrich) for $20 \mathrm{~min}$. Microtubules were stained with primary mouse antibodies (Abs) anti- $\alpha$-tubulin (cat no. T5168; Sigma-Aldrich) for $30 \mathrm{~min}$ and secondary donkey anti-mouse IgG conjugated with rhodamine Red-X (cat. no. 715-025-151; Jackson ImmunoResearch Laboratories) for $30 \mathrm{~min}$. Viral antigens were stained with rabbit anti-ECTV Abs conjugated with FITC for $60 \mathrm{~min}$ [8]. Nuclear and viral DNA was stained with Hoechst 33342 (Sigma-Aldrich) for $15 \mathrm{~min}$. After staining coverslips were mounted using ProLong Gold Antifade Reagent (Invitrogen).

\section{Fluorescence microscopy}

Slides were examined using fluorescence microscopes (Olympus BX60 and Olympus IX71) equipped with a Color View III cooled CCD camera. Cell ${ }^{\wedge} \mathrm{F}$ (Olympus) and ImageJ (NIH) software were used to analyze the images. CellSens Dimension (Olympus) was used for constrained iterative deconvolution. 


\section{Scanning electron microscopy (SEM)}

RAW 264.7 macrophages or GM-BM grown on microscopic slides were fixed for 60 min with $2.5 \%$ glutaraldehyde in phosphate buffer and later post-fixed for $60 \mathrm{~min}$ with $1 \%$ osmium tetroxide in phosphate buffer and dehydrated in ethanol and acetone series. The specimens were then dried using a CPD 7501 critical point drier (Polaron), coated with a gold layer in a JFC-1300 sputter-coater (JEOL) and analyzed using an FEI Quanta 200 environmental scanning electron microscope (ESEM) with the EDAX EDS system (FEI).

\section{Results}

\section{ECTV induces formation of long cellular extensions in RAW 264.7 macrophages and GM-BM cells}

In view of the importance of actin-based intracellular transport and intercellular spread regarding viral virulence, in this study we characterized cellular alteration in ECTV infected RAW 264.7 macrophages and GM-BM. Uninfected RAW 264.7 cells exhibited a round shape (Fig. 1A) or were slightly elongated (Fig. 1B) and showed the presence of cellular extensions. SEM analysis revealed membrane ruffles on the dorsal surface of the uninfected macrophages (Fig. 1C). Meanwhile, infected RAW 264.7 cells lost their normal shape and exhibited the presence of multiple long cellular extensions, whose length often exceeded the diameter of the cell (Figs. 1D and 1E). Morphology of long extensions varied greatly; some were straight or bent, and others branched in different directions. The long cellular protrusions were of variable length and at times formed a convex structure resembling a "bubble" (Fig. 1F). Some "bubbles" were found to be covered by numerous viral particles (Fig. 1G). SEM analysis revealed that ECTV particles associated with the surface membrane of bubble-like structures (Fig. 1G), and probably progeny virions may escape from the cell within these structures and may be released from their surface $[12,13]$. In addition to formation of long cellular extensions, infected RAW 264.7 macrophages at 24 hours post-infection (hpi) also showed cell surface flattening and loss of ruffles (Fig. 1G).

Comparable morphological changes were observed in ECTV-infected GM-BM. Uninfected GM-BM exhibited a round shape with the presence of characteristic dendritic projections and veils (Figs. 2A and 2B). At 24 hpi with ECTV GM-BM also showed the presence of multiple long and irregular cellular extensions, which gave the cells a unique, multifaceted morphology (Figs. 2C and 2D).

\section{Long actin-based extensions contain mitochondria and microtubules}

To better characterize ECTV-induced long cellular projections, we performed fluorescence staining of F-actin filaments using TRITC- or FITC-conjugated phalloidin (Figs. 3 and 4). Fig. 3A shows evidence that control RAW 264.7 macrophages were not infected with ECTV, and show only nuclear DNA. Cellular projections formed by ECTV-infected cells were composed of F-actin and were of different width: some were thick (width $=0.45 \mu \mathrm{m}$ ) (Fig. 3B), and others were much wider (width $=1.55 \mu \mathrm{m}$ ) (Fig. 4B) and could additionally expand or narrow at various lengths (Fig. 4B). Moreover, some of these projections were expanded on their ends and formed bubble-like structures (Figs. 1F and 3B). In these expanded extensions numerous viral particles occurred singly or in clusters (Fig. 3B), confirmed by immunofluorescence microscopy by visualization of viral DNA (labeled with Hoechst 33342) co-localization with viral antigen (stained by Abs anti-ECTV). Uninfected RAW 264.7 macrophages and GM-BM were devoid of such long actin-based projections (Figs. 3A and 4A).

The interaction between cytoskeletal proteins and mitochondria is important for maintenance of mitochondrial and cellular physiology. For that reason we assessed the mitochondrial organization within long cellular extensions. Within ECTV-induced long actin-based projections, mitochondria usually occurred separately as small, discrete organelles, which accumulated in the expanded regions of the extensions. In such regions, progeny viral particles were also observed (Fig. 4B). Moreover, the accumulation of mitochondria was additionally observed at the ends of long cellular extensions (Fig. 4B), especially when they were connected with neighboring cells (Fig. 5B).

Our last question concerned the distribution of microtubules in ECTV-induced long cellular extensions. In uninfected RAW 264.7 macrophages (Fig. 5A) the microtubule network was composed of wavy and intertwined filaments that branched towards the cell periphery. A similar pattern of microtubule network organization was detected in uninfected GM-BM (data not shown). ECTV-induced long cellular extensions contained a few ran along microtubule filaments, which were straighter, "relaxed," and less intertwined (Fig. 5B). Around microtubules many punctuate mitochondria were localized. Within bubble-like structures, containing numerous progeny virions, tubulin filaments were separated from each other and formed scaffolds for accumulated mitochondria (Fig. 5B).

\section{Discussion}

Macrophages and DCs represent major immune cells that are at the front line of defense against pathogens, including viruses. ECTV can infect splenic DCs and macrophages and such infection can be persistent after acute mousepox in BALB/c mice [14]. Our previous studies have shown that ECTV is able to productively infect RAW 264.7 macrophages [15] and GM-BM [11]. At the early stage (4 hpi) of infection GM-BM displayed only the 

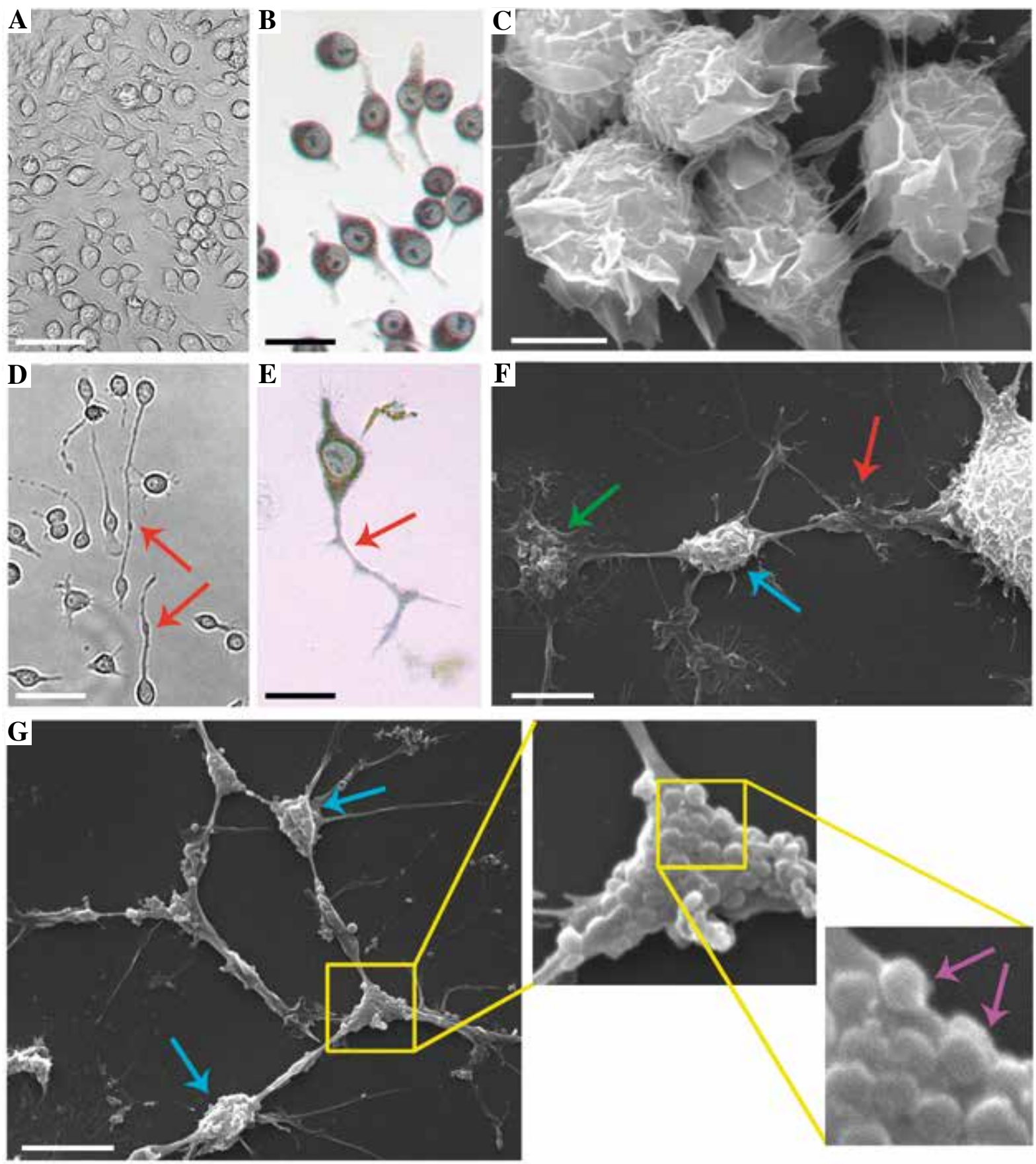

Fig. 1. Morphology of uninfected (A-C) and ECTV-infected (D-G) RAW 264.7 macrophages at 24 hours post-incubation with culture medium or $24 \mathrm{hpi}$, respectively. Micrographs from inverted microscope of unstained (A, D) and May-Grünwald-Giemsa stained $(\mathbf{B}, \mathbf{E})$ cells. Scanning electron micrographs of RAW 264.7 macrophages $(\mathbf{C}, \mathbf{F}, \mathbf{G})$. The magnified images are of the boxed regions. Arrows indicate: long cellular extensions (red), cytoplasmic packets (blue), extended end of long extension (green), viral particles (purple). Scale bars $=50 \mu \mathrm{m}(\mathbf{A}, \mathbf{D}), 25 \mu \mathrm{m}(\mathbf{B}, \mathbf{E})$ and $5 \mu \mathrm{m}(\mathbf{C}, \mathbf{F}, \mathbf{G})$

presence of virus replication centers termed "viral factories", whereas at the late stage ( $24 \mathrm{hpi}$ ) of infection, upon completion of viral morphogenesis, progeny virions were found near the plasma membrane and within cellular ex- tensions formed at this time post-infection [11]. Due to the presence of multiple long cellular extensions GM-BM adopted a unique morphology, previously observed in L929 fibroblasts infected with ECTV [8]. 

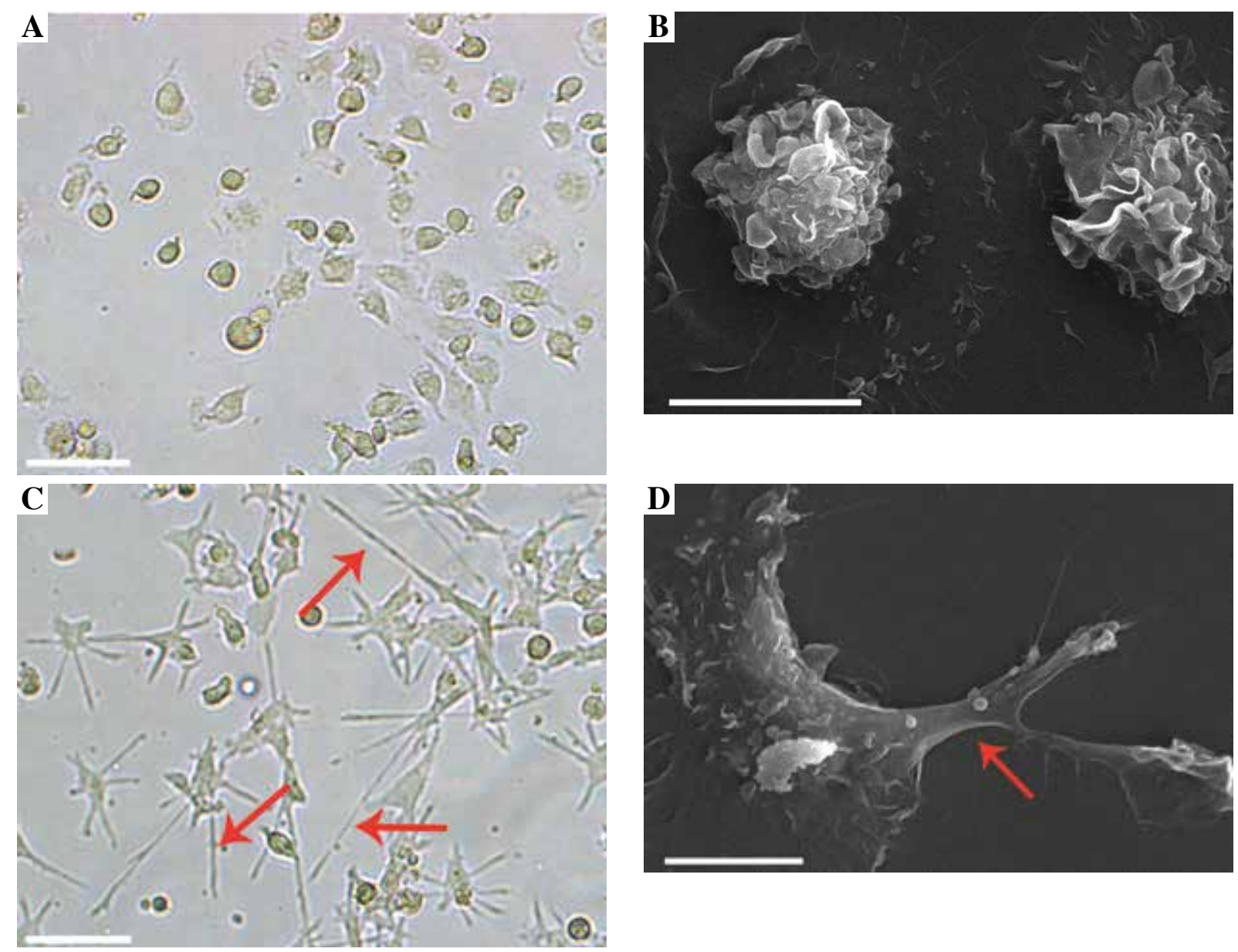

Fig. 2. Morphology of uninfected (A, B) and ECTV-infected (C, D) GM-BM at 24 hours post-incubation with culture medium or 24 hpi, respectively. Micrographs from inverted (A, C) and scanning electron (B, D) microscope. Red arrows indicate long cellular extensions. Scale bars $=50 \mu \mathrm{m}(\mathbf{A}, \mathbf{C})$ and $10 \mu \mathrm{m}(\mathbf{B}, \mathbf{D})$

In the present study we observed that long actin-based projections formed by ECTV-infected macrophages and DCs could extend and form convex structure resembling a "bubble". Such bubble-like structures were previously found in African green monkey kidney (BSC-1) cells infected with VACV at 20 hpi [12] and human amnion (FL) cells infected with the IHD-W strain of VACV at 48 hpi [13], and were called "cytoplasmic packets". A previous study showed that VACV could be released from cytoplasmic packets via "budding" [13]. Transmission electron microscopy (TEM) analysis has documented that cytoplasmic packets are sites where naked VACV particles are wrapped in cell surface membrane and are then released from the cell surface [13]. Cytoplasmic packets containing multiple virions allow massive release of progeny virus from the cell periphery $[12,13]$.

Within long cellular protrusions mitochondria occurred as small, discrete organelles, which accumulated in the cytoplasmic packets and at the ends of actin-based protrusions. It is not excluded that long projections, induced by ECTV, allow the free flow of the cytoplasm to neighboring cells, together with mitochondria and virions. It has been demonstrated that intercellular communication between the cells is possible through actin-based filopodial bridges, known as TNTs or cytonemes [16]. Some TNTs bearing microtubules are capable of transporting organelles, such as mitochondria, lysosomes and lipid droplets [17]. HIV-infected human macrophages have been shown to form thin, actin-based TNTs, which allowed spreading of HIV particles toward the uninfected cells [18]. Also, influenza virus exploits TNTs to transfer viral proteins and genomes from infected to uninfected, neighboring cells, resulting in productive viral replication in the recipient cell [19]. Interestingly, TNTs provide an alternative route for delivery of oncolytic viruses and of viral thymidine kinase activated drugs to amplify the bystander effect between cancer cells over long distances in the stroma-rich tumor microenvironment [20]. 

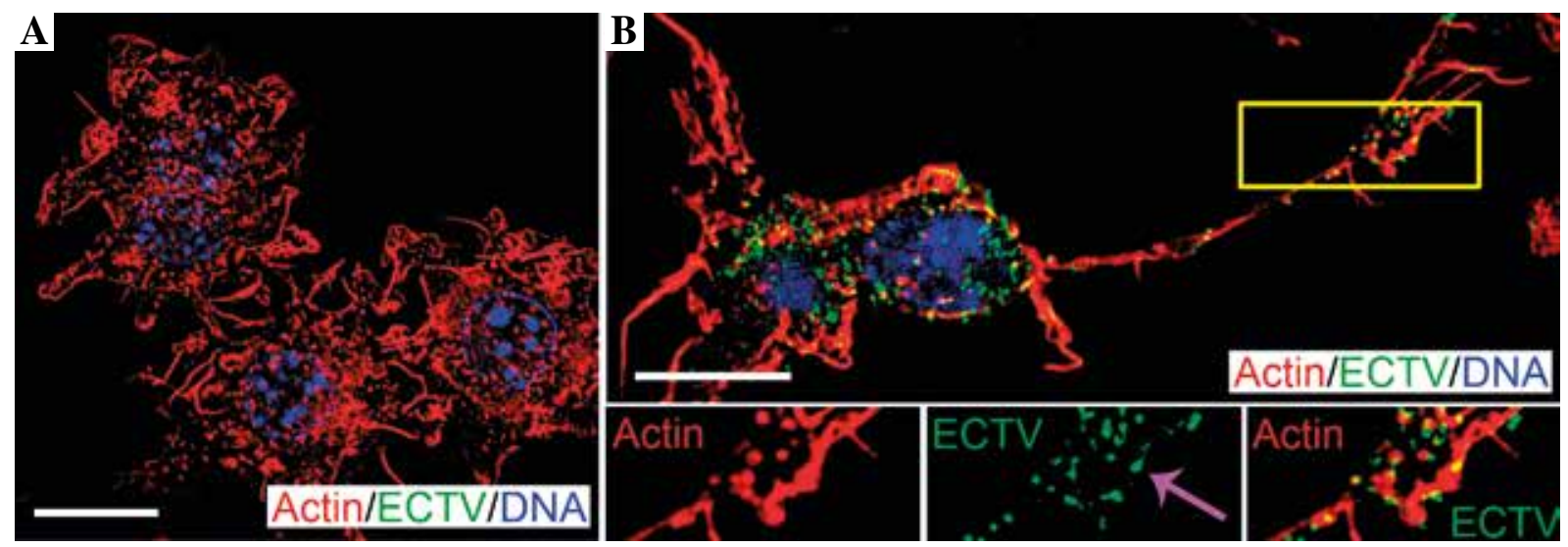

Fig. 3. Fluorescence microscopy analysis of actin cytoskeleton in uninfected (A) and ECTV-infected (B) RAW 264.7 macrophages at 24 hours post-incubation with culture medium or $24 \mathrm{hpi}$, respectively. The magnified images are of the boxed regions. Arrow indicates: viral antigen or DNA (purple). Scale bars $=10 \mu \mathrm{m}$
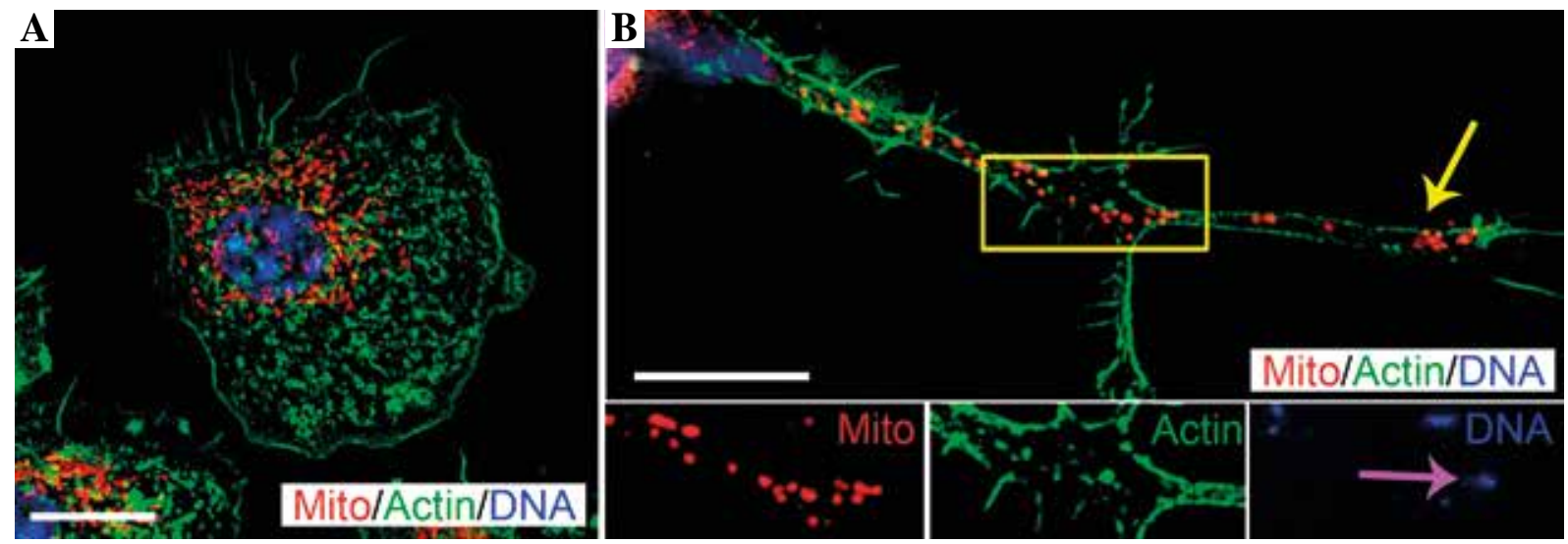

Fig. 4. Fluorescence microscopy analysis of actin and mitochondria distribution in uninfected (A) and ECTV-infected (B) GM-BM at 24 hours post-incubation with culture medium or $24 \mathrm{hpi}$, respectively. The magnified images are of the boxed regions. Arrows indicate: accumulation of mitochondria at the end of extension (yellow), viral antigen or DNA (purple). Scale bars $=10 \mu \mathrm{m}$
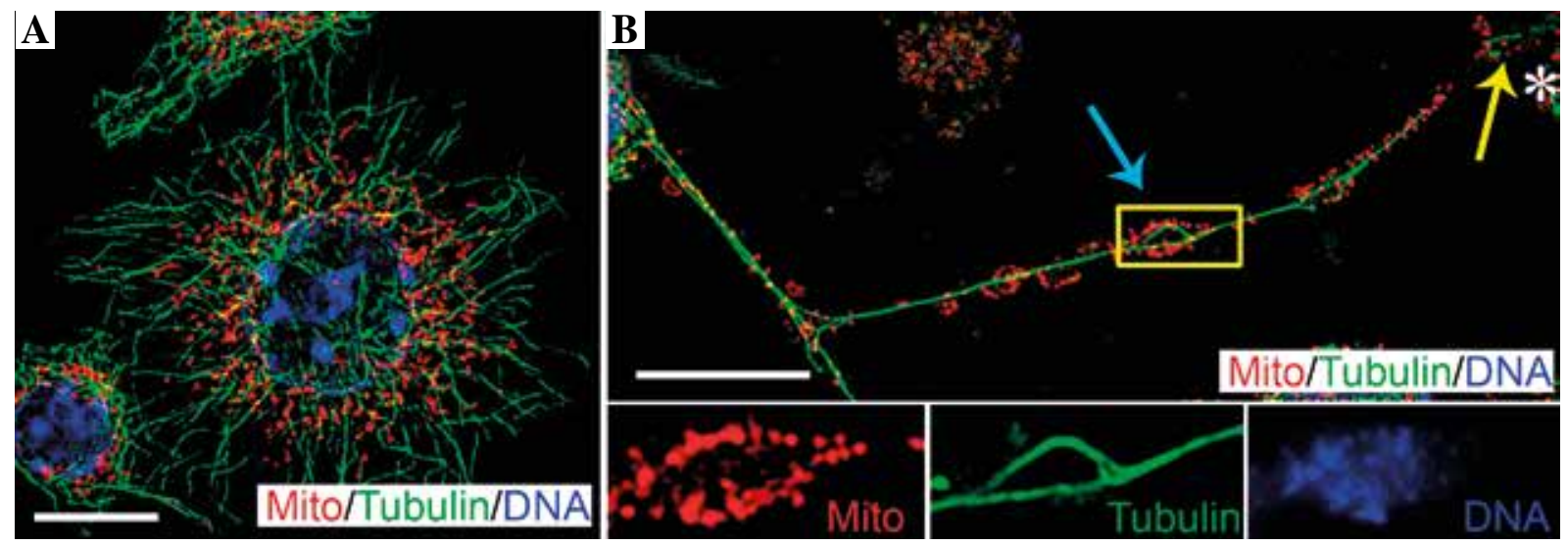

Fig. 5. Fluorescence microscopy analysis of tubulin and mitochondria distribution in uninfected (A) and ECTV-infected (B) RAW 264.7 macrophages at 24 hours post-incubation with culture medium or $24 \mathrm{hpi}$, respectively. The magnified images are of the boxed regions. Arrows indicate: bubble-like structure (blue), accumulation of mitochondria at the end of extension (yellow). Asterisk indicates neighboring cell. Scale bars $=10 \mu \mathrm{m}$ 
Accumulation of mitochondria within cytoplasm packets has never been demonstrated before. It is known that mitochondria supply the energy required for the virus morphogenetic processes, and a massive migration of the organelle to the virus assembly sites has been observed in permissive cells infected with African swine fever virus (ASFV) [21], rubella virus (RV) [22] and ECTV [23]. However, at the moment, we do not know the biological significance of mitochondria accumulation within actin-based structures together with newly assembled progeny virions that are "ready" to be released from the cell through budding. It has been shown that fragmentation of the mitochondrial network and formation of small, punctuate organelles facilitate their transport along microtubules [24]. Microtubules via motor proteins participate in the long distance mitochondrial transport, whereas the actin cytoskeleton anchors mitochondria and is important for short-range mitochondrial movement [25]. Therefore, it is not excluded that progeny virions exploit discrete mitochondria for efficient transport along the cytoskeletal components to the sites of viral budding. This statement, however, requires justification in further investigation.

In conclusion, our results showed the formation of long actin-based cellular protrusions by ECTV-infected macrophages and DCs. Such extensions have similar features as those of "cytoplasmic corridors", previously observed in rabbit kidney cells (RK13) infected with myxoma virus (MYXV) [26, 27] or VACV [27], and L929 fibroblasts infected with ECTV [8]. Cytoplasmic corridors participate in transportation of progeny virions to neighboring cells and promote virus spread $[8,26,27]$. Presumably, long extensions observed in ECTV-infected macrophages and DCs also support viral dissemination. On the other hand, morphological changes that infected macrophages and DCs undergo may interfere with their interaction with responder $\mathrm{T}$ cells and inhibit activation of the latter. Taken together, our study shows that ECTV exhibits remarkable ability to adapt to its natural host immune cells, in which it can replicate productively [28] and induce actin-based mechanisms for efficient viral spread and dissemination in vitro.

\section{Acknowledgments}

This work was supported by grants No. UMO2011/03/B/NZ6/03856 and UMO-2012/05/D/NZ6/02916 from the National Science Center in Cracow, Poland.

The authors declare no conflict of interest.

\section{References}

1. Taylor MP, Koyuncu OO, Enquist LW (2011): Subversion of the actin cytoskeleton during viral infection. Nat Rev Microbiol 9: 427-439.
2. Panchanathan V, Chaudhri G, Karupiah G (2006): Protective immunity against secondary poxvirus infection is dependent on antibody but not on CD4 or CD8 T-cell function. J Virol 80: 6333-6338.

3. Shchelkunov SN (2013): An increasing danger of zoonotic orthopoxvirus infections. PLoS Pathog 9: e1003756.

4. Reynolds MG, Damon IK (2012): Outbreaks of human monkeypox after cessation of smallpox vaccination. Trends Microbiol, 20: 80-87.

5. Shchelkunov SN (2013): An increasing danger of zoonotic orthopoxvirus infections. PLoS Pathog 9: e1003756.

6. Boratyńska A, Martyniszyn L, Szulc L, et al. (2010): Contribution of rearranged actin structures to the spread of Ectromelia virus infectionin vitro. Acta Virol 54: 41-48.

7. Boratyńska A, Martyniszyn L, Szulc L, et al. (2011): Membrane bridges and their role in the spread of ectromelia virus in vitro. Medycyna Wet 67: 474-477.

8. Szulc-Dabrowska L, Gregorczyk KP, Struzik J, et al. (2016): Remodeling of the fibroblast cytoskeletal architecture during the replication cycle of ectromelia virus: A morphological in vitro study in a murine cell line. Cytoskeleton 73: 396-417.

9. Chen TR (1977): In situ detection of mycoplasma contamination in cell cultures by fluorescent Hoechst 33258 stain. Exp Cell Res 104: 255-262.

10. Lutz MB, Kukutsch N, Ogilvie AL, et al. (1999): An advanced culture method for generating large quantities of highly pure dendritic cells from mouse bone marrow. J Immunol Methods, 223: 77-92.

11. Szulc-Dąbrowska L, Struzik J, Ostrowska A, et al. (2017): Functional paralysis of GM-CSF-derived bone marrow cells productively infected with ectromelia virus. PLoS ONE 12: e0179166.

12. Stokes GV (1976): High-voltage electron microscope study of the release of vaccinia virus from whole cells. J Virol 18: 636-643.

13. Tsutsvi K, Uno F, Akatsuka K, et al. (1983): Electron microscopic study on vaccinia virus release. Arch Virol 75: 213218.

14. Spohr de Faundez I, Gieryńska M, Niemiałtowski M G, et al. (1995): Ectromelia virus establishes a persistent infection in spleen dendritic cells and macrophages of BALB/c mice following the acute disease. Adv Exp Med Biol 378: 257-261.

15. Martyniszyn L, Szulc-Dąbrowska L, Boratyńska-Jasińska A, et al. (2013): Crosstalk between autophagy and apoptosis in RAW 264.7 macrophages infected with ectromelia orthopoxvirus. Viral Immunol 26: 322-335.

16. Gurke S, Barroso JFV, Gerdes HH (2008): The art of cellular communication: tunneling nanotubes bridge the divide. Histochem Cell Biol 129: 539-550.

17. Tang BL (2016): MIRO GTPases in mitochondrial transport, homeostasis and pathology. Cells 5: 1.

18. Eugenin EA, Gaskill PJ, Berman JW (2009): Tunneling nanotubes (TNT) are induced by HIV-infection of macrophages: a potential mechanism for intercellular HIV trafficking. Cell Immunol 254: 142-148.

19. Kumar A, Kim JH, Ranjan P, et al. (2017): Influenza virus exploits tunneling nanotubes for cell-to-cell spread. Sci Rep 7: 40360 .

20. Ady J, Thayanithy V, Mojica K, et al. (2016): Tunneling nanotubes: an alternate route for propagation of the bystander effect following oncolytic viral infection. Mol Ther Oncolytics 3: 16029. 
21. Rojo G, Chamorro M, Salas ML, et al. (1998): Migration of mitochondria to viral assembly sites in African swine fever virus-infected cells. J Virol 72: 7583-7588.

22. Lee JY, Bowden DS (2000): Rubella virus replication and links to teratogenicity. Clin Microbiol Rev 13: 571-587.

23. Gregorczyk KP, Szulc-Dąbrowska L, Wyżewski Z, et al. (2014): Changes in the mitochondrial network during ectromelia virus infection of permissive L929 cells. Acta Biochim Pol 61: 171-177.

24. Varadi A, Johnson-Cadwell LI, Cirulli V, et al. (2004): Cytoplasmic dynein regulates the subcellular distribution of mitochondria by controlling the recruitment of the fission factor dynamin-related protein-1. J Cell Sci 117: 4389-400.

25. MacAskill AF, Kittler JT (2010): Control of mitochondrial transport and localization in neurons. Trends Cell Biol 20: 102-112.

26. Duteyrat JL, Gelfi J, Bertagnoli S (2006): Ultrastructural study of myxoma virus morphogenesis. Arch Virol 151: 2161-2180.

27. Irwin CR, Evans DH (2012): Modulation of the myxoma virus plaque phenotype by vaccinia virus protein F11. J Virol 86: 7167-7179.

28. Szulc-Dąbrowska L, Struzik J, Cymerys J, et al. (2017): The in vitro inhibitory effect of ectromelia virus infection on innate and adaptive immune properties of GM-CSF-derived bone marrow cells is mouse strain-independent. Front Microbiol 8: 2539. 